\title{
GENERAL CONVOLUTIONS OF INTEGRAL TRANSFORMS AND THEIR APPLICATION TO ODE AND PDE PROBLEMS
}

\section{L.E. BRITVINA}

Department of Theoretical and Mathematical Physics, Novgorod State University

ul.St.Petersburgskaya 41, Novgorod the Great, 173003, Russia

E-mail: bmv@novsu.ac.ru

Received October 15, 2005; revised February 5, 2006

Abstract. The present research is devoted to some polyconvolutions generated by various integral transforms. For example, we study convolutions of the Hankel transform with the following factorization properties:

$$
\begin{aligned}
& \mathrm{H}_{\nu}[h](x)=\mathrm{H}_{\mu}[f](x) \mathrm{H}_{\mu+\nu}[g](x), \quad \mathrm{H}_{\nu+m}[h](x)=x^{-\nu} \mathrm{H}_{\nu+m}[f](x) \mathrm{H}_{\nu}[g](x), \\
& \mathrm{H}_{\nu}[h](x)=x^{-\nu} \mathrm{H}_{\mu}[f](x) \mathrm{H}_{\mu}[g](x), \quad \mathrm{H}_{\mu}[h](x)=x^{-\nu} \mathrm{H}_{\nu}[f](x) \mathrm{H}_{\mu}[g](x),
\end{aligned}
$$

where $\mathrm{H}_{\nu}[f](x)$ is the Hankel transform. Conditions for the existence of the constructed polyconvolutions are found. The results of this research are applied for solvability of ODEs and PDEs by the method of integral transforms. The derived constructions allow us to solve various nonuniform equations.

Key words: integral transforms, convolution, Hankel transform, ODE, PDE

\section{Introduction}

The Hankel transform is the most extensively studied area of the theory of Bessel transforms. This transform is used to solve many problems of mathematical physics. It is defined by the integral

$$
F_{\nu}(u)=\mathrm{H}_{\nu}[f](u)=\int_{0}^{\infty} f(t) t J_{\nu}(u t) d t, \quad x \in \mathbf{R}_{+},
$$

where $J_{\nu}(z)$ is the Bessel function of the first kind of order $\nu, \operatorname{Re} \nu>-1 / 2$.

With the help of the definition of polyconvolution, or generalized convolution, we can construct various polyconvolutions generated by transform (1.1). 
This definition was first introduced by V.A. Kakichev in 1967 [8]. This is the key definition of the present research.

Definition 1. Let $A_{1}, A_{2}$ and $A_{3}$ be operators. The generalized convolution of function $f(t)$ and $g(t)$, under $A_{1}, A_{2}, A_{3}$, with weighted function $\alpha(x)$, is the function $h(t)$ denoted by $\left(f_{A_{1}} \stackrel{*}{*} g_{A_{2}}\right)_{A_{3}}(t)$ for which the following factorization property is valid:

$$
\left(A_{3} h\right)(x)=A_{3}\left[\left(f_{A_{1}} \stackrel{\alpha}{*} g_{A_{2}}\right)_{A_{3}}\right](x)=\alpha(x)\left(A_{1} f\right)(x)\left(A_{2} g\right)(x) .
$$

In case of the Hankel transform we denote polyconvolution by $\left(f_{\mu} \stackrel{\alpha}{*} g_{\eta}\right)_{\nu}(t)$ if it satisfies the factorization property

$$
\mathrm{H}_{\nu}[h](x)=\mathrm{H}_{\nu}\left[\left(f_{\mu}^{*} * g_{\eta}\right)_{\nu}\right](x)=x^{\alpha} \mathrm{H}_{\mu}[f](x) \mathrm{H}_{\eta}[g](x) .
$$

The classical convolution of the Hankel transform was first introduced by Ya.I. Zhitomirskii in 1955 [16]. In 1967 V.A. Kakichev constructed this convolution with help of definition [8].

The explicit expression of this convolution is

$$
\begin{aligned}
\left(f_{\nu} \stackrel{-\nu}{*} g_{\nu}\right)_{\nu}(t)= & \frac{t^{\nu}}{2^{\nu} \sqrt{\pi} \Gamma(\nu+1 / 2)} \int_{0}^{\pi} \sin ^{2 \nu} s d s \int_{0}^{\infty} f(\tau) \\
& \times \frac{g\left(\sqrt{t^{2}+\tau^{2}-2 t \tau \cos s}\right)}{\left(t^{2}+\tau^{2}-2 t \tau \cos s\right)^{\nu / 2}} \tau^{\nu+1} d \tau .
\end{aligned}
$$

The Hankel transform is the Mellin convolution type transform. There are many works, which are devoted to study of these transforms and their convolutions (see, for example, $[7,13,15])$. A number of convolution constructions involving the Hankel transform was derived by N.X.Thao and N.T.Xai [12]. Some polyconvolutions obtained by the author with the help of an approach by V.A. Kakichev were given in [9].

\section{The Generalized Convolutions of Hankel Transform}

The polyconvolutions with the factorization properties

$$
\mathrm{H}_{\nu}[h](x)=x^{-\nu} \mathrm{H}_{\mu}[f](x) \mathrm{H}_{\mu}[g](x), \quad \mathrm{H}_{\mu}[h](x)=x^{-\nu} \mathrm{H}_{\nu}[f](x) \mathrm{H}_{\mu}[g](x) .
$$

were introduced in [4]. Let us consider the functions 


$$
\begin{aligned}
& h_{1}(t)=t^{-\nu} \iint_{|u-v|<t<u+v} u^{\nu} f(u) g(v) P_{1}(t ; u, v) d u d v \\
& -t^{-\nu} \int_{0}^{\infty} d u \int_{0}^{t-u} u^{\nu} f(u) g(v) Q_{1}(t ; u, v) d v \\
& h_{2}(t)=t^{\nu-1} \int_{0}^{\infty} d v \int_{|t-v|}^{t+v} u^{-\nu+1} f(u) g(v) P_{2}(t ; u, v) d u \\
& -t^{\nu-1} \int_{0}^{\infty} d v \int_{t+v}^{\infty} u^{-\nu+1} f(u) g(v) Q_{2}(t ; u, v) d u
\end{aligned}
$$

where

$$
\begin{aligned}
& P_{i}(t ; u, v)=\frac{1}{\sqrt{2 \pi}} v^{\nu} P_{\mu-1 / 2}^{1 / 2-\nu}\left(\cos s_{i}\right) \sin ^{\nu-1 / 2} s_{i}, \\
& Q_{i}(t ; u, v)=\frac{\sqrt{2}}{\pi^{3 / 2}} \sin [(\mu-\nu) \pi] \mathrm{e}^{\frac{(2 \nu-1) \pi}{2 i}} v^{\nu} Q_{\mu-1 / 2}^{1 / 2-\nu}\left(\operatorname{ch} r_{i}\right) \operatorname{sh}^{\nu-1 / 2} r_{i},
\end{aligned}
$$

$i=1,2, P_{\mu}^{\nu}(x), Q_{\mu}^{\nu}(x)$ are the associated Legendre functions of the first and second kind, respectively, and

$$
\begin{aligned}
& 2 u v \cos s_{1}=u^{2}+v^{2}-t^{2}, \quad 2 u v \operatorname{ch} r_{1}=t^{2}-u^{2}-v^{2}, \\
& 2 t v \cos s_{2}=t^{2}+v^{2}-u^{2}, \quad 2 t v \operatorname{ch} r_{2}=u^{2}-t^{2}-v^{2} .
\end{aligned}
$$

Consider the following theorems which present the conditions of existence of polyconvolutions (2.2) and (2.3).

Theorem 1. Suppose that $\sqrt{t} f(t), \sqrt{t} g(t) \in L(0, \infty)$ and $\operatorname{Re} \nu>1 / 2$, $\operatorname{Re} \mu>(2 \operatorname{Re} \nu-3) / 4$. Then the function $h_{1}(t)$ exists and the following factorization relation is valid.

$$
\mathrm{H}_{\nu}\left[h_{1}\right](x)=x^{-\nu} \mathrm{H}_{\mu}[f](x) \mathrm{H}_{\mu}[g](x) .
$$

Proof. It is well known that [10]

$$
\int_{0}^{\infty} x^{1-\nu} J_{\nu}(x t) J_{\mu}(x u) J_{\mu}(x v) d x=I,
$$

where $\operatorname{Re} \mu>-1, \operatorname{Re} \nu>-1 / 2$,

$$
I=\left\{\begin{array}{l}
0, \quad 0<t<|u-v|, u, v>0, \\
\frac{u^{\nu-1}}{v t^{\nu}} P_{1}(t ; u, v), \quad|u-v|<t<u+v, u, v>0, \\
-\frac{u^{\nu-1}}{v t^{\nu}} Q_{1}(t ; u, v), \quad t>u+v, \quad u, v>0
\end{array}\right.
$$


and the functions $P_{1}(t ; u, v)$ and $Q_{1}(t ; u, v)$ are defined by relations (2.4) and (2.5), respectively.

Using the asymptotic expansion of Bessel functions of the first kind (see, for example, [1]), we can easily show that there exists a positive number $C_{1}$ independent of $t \in(0, \infty)$ such that

$$
\left|\sqrt{t} J_{\nu}(t)\right|<C_{1}, \quad \forall t \in(0, \infty)
$$

and $t^{-\nu} J_{\nu}(t) \in L(0, \infty)$, for $\operatorname{Re} \nu>1 / 2$. Therefore,

$$
\begin{gathered}
\left|\int_{0}^{N} x^{1-\nu} J_{\nu}(x t) J_{\mu}(x u) J_{\mu}(x v) d x\right| \leq \frac{C_{1}^{2}}{\sqrt{u v}} \int_{0}^{N}\left|x^{-\nu} J_{\nu}(x t)\right| d x \\
\leq \frac{C_{1}^{2} t^{\operatorname{Re} \nu-1}}{\sqrt{u v}} \int_{0}^{\infty}\left|x^{-\nu} J_{\nu}(x)\right| d x \leq \frac{C t^{\operatorname{Re} \nu-1}}{\sqrt{u v}},
\end{gathered}
$$

where $C$ is independent of $t, u, v$ and $N$.

Since $\sqrt{t} f(t), \sqrt{t} g(t) \in L(0, \infty)$, we obtain

$$
\left|h_{1}(t)\right| \leq C t^{\operatorname{Re} \nu-1} \int_{0}^{\infty} \int_{0}^{\infty} \sqrt{u v} f(u) g(v) d u d v<\infty .
$$

Thus, the function $h_{1}(t)$ defined by expression (2.2) exists. Let us now prove the validity of factorization relation (2.1). It follows from (2.8) and (2.9) that the Lebesgue theorem on the convergence under the sign of the integral is applicable in our case and

$$
\begin{aligned}
h_{1}(t) & =\int_{0}^{\infty} \int_{0}^{\infty} u v f(u) g(v) \int_{0}^{\infty} x^{1-\nu} J_{\nu}(x t) J_{\mu}(x u) J_{\mu}(x v) d x d u d v \\
& =\int_{0}^{\infty} x J_{\nu}(x t) x^{-\nu} \mathrm{H}_{\mu}[f](x) \mathrm{H}_{\mu}[g](x) d x .
\end{aligned}
$$

Here we have also used the theorem on the existence of the Hankel transform of the function $f(t)$ belonging to $L(0, \infty)$ with weight $\sqrt{t}$ (see [13]). It is well known that

$$
\mathrm{H}_{\mu}[f](x)=O\left(x^{\mu}\right), \quad x \rightarrow+0,
$$

for $\sqrt{t} f(t)$ belonging to $L(0, \infty)$ (see [6]). Therefore,

$$
H(x)=x^{-\nu} \mathrm{H}_{\mu}[f](x) \mathrm{H}_{\mu}[g](x)=O\left(x^{2 \mu-\nu}\right), \quad x \rightarrow+0 .
$$

On the other hand, applying inequality (2.7) and taking $\sqrt{t} f(t) \in L(0, \infty)$ into account, we obtain 


$$
\left|\mathrm{H}_{\mu}[f](x)\right| \leq \frac{1}{\sqrt{x}} \int_{0}^{\infty}|\sqrt{t} f(t)|\left|\sqrt{x t} J_{\mu}(x t)\right| d t \leq \frac{C_{1}}{\sqrt{x}}, \quad x \in(0, \infty),
$$

where $C_{1}$ is independent of $x$ and $\mu$.

Similarly, $\mathrm{H}_{\mu}[g](x)=O\left(x^{-1 / 2}\right)$. Thus, $H(x)=O\left(x^{-\nu-1}\right)$ for all $x \in \mathbf{R}_{+}$. In particular,

$$
H(x)=O\left(x^{-\nu-1}\right), \quad x \rightarrow+\infty .
$$

Therefore, for $\operatorname{Re} \nu>1 / 2$ and $\operatorname{Re} \mu>(2 \operatorname{Re} \nu-3) / 4$, it follows from (2.11) and (2.12) that $\sqrt{x} H(x) \in L(0, \infty)$; formula $(2.10)$ can be rewritten as $h_{1}(t)=$ $\mathrm{H}_{\nu}^{-1}[H(x)](t)$.

Further, applying the theorem on the inversion of the Hankel transform in the class $L(0, \infty)$ with weight $\sqrt{x}$ (see [13]), we can write the function $H(x)$ as follows:

$$
H(x)=\mathrm{H}_{\nu}\left[h_{1}(t)\right](x) .
$$

Formulas (2.11) and (2.13) yield factorization relation (2.1). The theorem is proved.

The following assertion can be proved in a similar way.

Theorem 2. Suppose that $\sqrt{t} f(t), \sqrt{t} g(t) \in L(0, \infty)$ and $\operatorname{Re} \nu>1 / 2$, $\operatorname{Re} \mu>\operatorname{Re} \nu-1$. Then the function $h_{2}(t)$ exists and the following factorization relation is valid

$$
\mathrm{H}_{\mu}\left[h_{2}\right](x)=x^{-\nu} \mathrm{H}_{\mu}[f](x) \mathrm{H}_{\mu}[g](x) .
$$

The other convolution construction generated by the Hankel transform is the polyconvolution with the factorization property

$$
\mathrm{H}_{\nu+m}[h](x)=x^{-\nu} \mathrm{H}_{\nu+m}[f](x) \mathrm{H}_{\nu}[g](x) .
$$

In this case we prove the following theorem [5].

Theorem 3. Suppose that $\sqrt{t} f(t), \sqrt{t} g(t) \in L(0, \infty), g(t)$ is the continuous function with a bounded variation on any interval $(0, R)$ and $\operatorname{Re} \nu>1 / 2$. Then the polyconvolution

$$
\begin{aligned}
h(t)=\frac{2^{\nu-1} t^{\nu} m ! \Gamma(\nu)}{\pi \Gamma(2 \nu+m)} \int_{0}^{\pi} C_{m}^{\nu}(\cos s) \sin ^{2 \nu} s \\
\quad \times \int_{0}^{\infty} f(\tau) \frac{g\left(\sqrt{t^{2}+\tau^{2}-2 t \tau \cos s}\right)}{\left(t^{2}+\tau^{2}-2 t \tau \cos s\right)^{\frac{\nu}{2}}} \tau^{\nu+1} d \tau,
\end{aligned}
$$

exists and factorization relation (2.15) is valid. Here $C_{m}^{\nu}(z)$ is Gegenbauer's polynomial. 
Proof. The function

$$
H(x)=x^{-\nu} \mathrm{H}_{\nu+m}[f](x) \mathrm{H}_{\nu}[g](x) .
$$

belongs to $L(0, \infty)$ with weight $\sqrt{t}$ for $\operatorname{Re} \nu>1 / 2$ (see [5]). The inequality $\operatorname{Re}(\nu+m)>-3 / 2$ is true for $\forall m \in \mathbf{N}_{0}$ and $\forall \nu, \operatorname{Re} \nu>1 / 2$. Therefore, the function

$$
h(t)=\mathrm{H}_{\nu+m}^{-1}[H(x)](t)
$$

exists and $h(t)$ is continuous and bounded for all $t \in \mathbf{R}_{+}$. We obtain

$$
\begin{aligned}
h(t) & =\int_{0}^{\infty} x^{1-\nu} \mathrm{H}_{\nu+m}[f](x) \mathrm{H}_{\nu}[g](x) J_{\nu+m}(x t) d x \\
& =\int_{0}^{\infty} \tau f(\tau) \int_{0}^{\infty} \mathrm{H}_{\nu}[g](x) x^{1-\nu} J_{\nu+m}(x t) J_{\nu+m}(x \tau) d x d \tau .
\end{aligned}
$$

It is known that [14]

$$
\begin{aligned}
J_{\nu+m}(a) J_{\nu+m}(b) & =\frac{2^{\nu-1} a^{\nu} b^{\nu} m ! \Gamma(\nu)}{\pi \Gamma(2 \nu+m)} \int_{0}^{\pi} C_{m}^{\nu}(\cos s) \sin ^{2 \nu} s \\
& \times \frac{J_{\nu}\left(\sqrt{a^{2}+b^{2}-2 a b \cos s}\right)}{\left(a^{2}+b^{2}-2 a b \cos s\right)^{\frac{\nu}{2}}} d s, \quad \operatorname{Re} \nu>-1 / 2 .
\end{aligned}
$$

Using formula (2.16) we find explicit form (2.16) for the polyconvolution $h(t)$. The theorem is proved.

Sometimes we need to introduce the differential operators [2]

$$
N_{m, \nu}=t^{\nu}\left(\frac{d}{t d t}\right)^{m} t^{m-\nu}, \quad S_{m, \nu}^{k}=\left[N_{m,-\nu} N_{m, \nu+m}\right]^{k},
$$

which allow us to find the conditions of existence of generalized convolutions.

These operators possess the following properties:

a) $S_{m, \nu}^{k}=S_{m,-\nu}^{k}=S_{k m, \nu}$,

where $S_{n, \nu}=S_{n, \nu}^{1}=S_{1, \nu}^{n}=\left[\frac{d^{2}}{d t^{2}}+\frac{1}{t} \frac{d}{d t}-\frac{\nu^{2}}{t^{2}}\right]^{n}$.

b) $N_{m, \pm \nu+k m} N_{m, \pm \nu+(k-1) m} \cdots N_{m, \pm \nu+m} N_{m, \pm \nu}=N_{m(k+1), \pm \nu+k m}$.

It should be noted that some special cases of these operators occur in the main equations of mathematical physics, for example, in elasticity theory. Using properties (a) and (b), it is readily verified that all well-know differential operators related to the Hankel transform can be expressed in terms of $N_{m, \pm \nu}$ and $S_{m, \nu}$. Therefore, we restrict ourselves to operators (2.17). 
Suppose that $C_{\lambda}(0, \infty)_{\rho}$ is the set of functions $f(t)$ with continuous derivatives on the interval $(0, \infty)$ such that the following asymptotic estimates hold [2]:

$$
f(t)=O\left(t^{\lambda}\right), t \rightarrow+0, \quad f(t)=O\left(t^{-\rho}\right), t \rightarrow+\infty .
$$

Further, $\rho, \lambda \in \mathbf{R}, \rho>3 / 2$ and $\lambda>-1 / 2$. In this case we can construct, for example, the polyconvolution with the factorization property

$$
\mathrm{H}_{\nu}[h](x)=\mathrm{H}_{\mu}[f](x) \mathrm{H}_{\mu+\nu}[g](x) .
$$

Theorem 4. Suppose that $\sqrt{t} f(t) \in L(0, \infty)$ and

$$
\begin{aligned}
& \sqrt{t} N_{2, \pm(\mu+\nu)+2} g(t) \in L(0, \infty), \quad N_{k, \pm(\mu+\nu)+k} g(t) \in C_{\lambda}(0, \infty)_{\rho}, \\
& k=0,1, \operatorname{Re} \mu>-1 / 2, \quad \operatorname{Re}(\mu+\nu)>-1 / 2+\max \{0, \mp 2\},
\end{aligned}
$$

then the polyconvolution

$$
\begin{gathered}
h(t)=\frac{2^{\mu+\nu} t^{\nu}}{\pi} \int_{-\pi / 2}^{\pi / 2} \mathrm{e}^{i(\mu-\nu) s} \cos ^{\mu+\nu} s \int_{0}^{\infty} f(\tau) g\left(\sqrt{2\left(\tau^{2} \mathrm{e}^{i s}+t^{2} \mathrm{e}^{-i s}\right) \cos s}\right) \\
\times\left(\tau^{2} \mathrm{e}^{i s}+t^{2} \mathrm{e}^{-i s}\right)^{-\frac{\mu+\nu}{2}} \tau^{\mu+1} d \tau d s
\end{gathered}
$$

exists and factorization relation (2.18) is valid.

Proof. Using the definition of polyconvolution and the definition of Hankel transform we obtain

$$
\begin{array}{r}
h(t)=\mathrm{H}_{\nu}^{-1}\left[\mathrm{H}_{\mu}[f](x) \mathrm{H}_{\mu+\nu}[g](x)\right](t)=\int_{0}^{\infty} \mathrm{H}_{\mu}[f](x) \mathrm{H}_{\mu+\nu}[g](x) x J_{\nu}(x t) d x \\
=\int_{0}^{\infty} \int_{0}^{\infty} f(\tau) \mathrm{H}_{\mu+\nu}[g](x) J_{\nu}(x t) J_{\mu}(x \tau) d x d \tau d x .
\end{array}
$$

Let us prove the existence of function $h(t)$.

$$
\begin{aligned}
|h(t)| & \leq \int_{0}^{\infty} d x \int_{0}^{\infty}|\sqrt{\tau} f(\tau)|\left|\sqrt{\tau x} J_{\mu}(x \tau)\right|\left|\sqrt{x t} J_{\nu}(t x)\right|\left|\frac{1}{\sqrt{t}} \mathrm{H}_{\mu+\nu}[g](x)\right| \\
& \leq \frac{C_{1}^{2}}{\sqrt{t}} \int_{0}^{\infty}\left|\mathrm{H}_{\mu+\nu}[g](x)\right| d x \int_{0}^{\infty}|\sqrt{\tau} f(\tau)| d \tau .
\end{aligned}
$$

Here we have used inequality (2.7). Function $\mathrm{H}_{\mu+\nu}[g](x)$ belongs to $L(0, \infty)$ with weight $\sqrt{t}$ (see [3]). Therefore,

$$
|h(t)| \leq \frac{C_{1}^{2}}{\sqrt{t}}\left\|\mathrm{H}_{\mu+\nu}[g](x)\right\|\|\sqrt{\tau} f(\tau)\|<\infty, t>0 .
$$


Thus, the polyconvolution $h(t)$ defined by expression (2.19) exist.

Let us now find the explicit form for the function $h(t)$. Using formula (2.12.27.10) from [10]:

$$
\begin{gathered}
J_{\mu}(a c) J_{\nu}(b c)=\frac{2^{\mu+\nu} a^{\mu} b^{\nu}}{\pi} \int_{-\pi / 2}^{\pi / 2} \mathrm{e}^{i(\mu-\nu) s} \cos ^{\mu+\nu} s[\varphi(s)]^{-\mu-\nu} J_{\mu+\nu}(c \varphi(s)) d s, \\
\operatorname{Re}(\mu+\nu)>-1, \quad a, b, c>0
\end{gathered}
$$

and the theorem on the inversion of the Hankel transform in the class $L(0, \infty)$ with weight $\sqrt{x}$ (see [3]), we obtain the explicit form (2.19) for the polyconvolution $h(t)$. The theorem is proved.

\section{Application of Polyconvolution to ODE and PDE Problems}

The results of this research are applied for solvability of ODE and PDE by the method of integral transforms [11]. The derived constructions allow us to solve various nonuniform equations.

Consider the equation

$$
\sum_{k} a_{k} \mathcal{L}^{k} u(t)=f(t)
$$

where $\mathcal{L}^{k}$ is a operator. For example,

$$
\mathcal{L}^{k} u(t)=\frac{d^{k} u(t)}{d t^{k}} \text { or } \mathcal{L}^{\mathrm{k}} \mathrm{u}(\mathrm{t})=\mathrm{u}\left(\mathrm{t}-\omega_{\mathrm{k}}\right)
$$

The solution of this equation can be represented as a polyconvolution

$$
u(t)=\left(\varphi_{B_{1}} * \psi_{B_{2}}\right)_{A}(t) .
$$

Example 1. Consider the equation (3.1) with the differential operators (2.17)

$$
\mathcal{L}^{k} h(t)=S_{k, \nu} h(t), \quad k=0,1,2, \ldots n, \quad t \in \mathbf{R}_{+}
$$

and zero conditions. Then

$$
\psi(t)=\mathrm{H}_{\nu}^{-1}[\Psi(x)], \quad \Psi(x)=x^{\nu} / \sum_{k=0}^{n}(-1)^{k} a_{k} x^{2 k}
$$

and the solution of this equation is given by

$$
u(t)=\left(f_{\nu} \stackrel{-\nu}{*} \psi_{\nu}\right)_{\nu}(t) .
$$

If $n=1, a_{0}=a, a_{1} \equiv 1,1 / 2<\operatorname{Re} \nu<3 / 2$, then we have the following equation 


$$
\frac{d^{2} u}{d t^{2}}+\frac{d u}{t d t}-\left(\frac{\nu^{2}}{t^{2}}-a\right) u(t)=f(t)
$$

Applying the results given above we obtain

$$
\psi(t)= \begin{cases}\frac{\pi}{2}|c|^{\nu} Y_{\nu}(|c| t), & \text { if } a=c^{2}, \\ -|c|^{\nu} K_{\nu}(|c| t), & \text { if } a=-c^{2},\end{cases}
$$

where $Y_{\nu}(z)$ and $K_{\nu}(z)$ are the Bessel function and the modified Bessel function of the second kind of order $\nu$, respectively. Then

$$
\begin{aligned}
u(t)=\frac{t^{\nu}|c|^{\nu} \sqrt{\pi}}{2^{\nu+1} \Gamma\left(\nu+\frac{1}{2}\right)} \int_{0}^{\pi} \sin ^{2 \nu} s d s \int_{0}^{\infty} f(\tau) & \\
\times & \frac{Y_{\nu}\left(|c| \sqrt{t^{2}+\tau^{2}-2 t \tau \cos s}\right)}{\left(t^{2}+\tau^{2}-2 t \tau \cos s\right)^{\nu / 2}} \tau^{\nu+1} d \tau, \quad a=c^{2},
\end{aligned}
$$

and

$$
\begin{aligned}
& u(t)=-\frac{t^{\nu}|c|^{\nu}}{2^{\nu} \sqrt{\pi} \Gamma\left(\nu+\frac{1}{2}\right)} \int_{0}^{\pi} \sin ^{2 \nu} s d s \int_{0}^{\infty} f(\tau) \\
& \times \frac{K_{\nu}\left(|c| \sqrt{t^{2}+\tau^{2}-2 t \tau \cos s}\right)}{\left(t^{2}+\tau^{2}-2 t \tau \cos s\right)^{\nu / 2}} \tau^{\nu+1} d \tau, \quad a=-c^{2} .
\end{aligned}
$$

Example 2. Let us consider the equation

$$
\frac{\partial^{2} v}{\partial t_{1}{ }^{2}}-b^{2}\left(\frac{\partial^{2} v}{\partial t_{2}^{2}}+\frac{1}{t_{2}} \frac{\partial v}{\partial t_{2}}\right)+\left(\gamma+\frac{b^{2} \nu^{2}}{t_{2}^{2}}\right) v\left(t_{1}, t_{2}\right)=f\left(t_{1}, t_{2}\right)
$$

with the following conditions

$$
u\left(+0, t_{2}\right)=u_{0}\left(t_{2}\right), \quad u_{t_{1}}^{\prime}\left(+0, t_{2}\right)=u_{1}\left(t_{2}\right) .
$$

The solution of this equation is given by

$$
\begin{aligned}
u\left(t_{1}, t_{2}\right) & =(f * \psi)_{\mathrm{L}, \nu}\left(t_{1}, t_{2}\right)+\left(u_{0} * \psi_{t_{1}}\right)_{\nu}\left(t_{1}, t_{2}\right)+\left(u_{1} * \psi\right)_{\nu}\left(t_{1}, t_{2}\right) \\
& =(f * \psi)_{\mathrm{L}, \nu}\left(t_{1}, t_{2}\right)+\frac{\partial}{\partial t_{1}}\left(u_{0} * \psi\right)_{\nu}\left(t_{1}, t_{2}\right)+\left(u_{1} * \psi\right)_{\nu}\left(t_{1}, t_{2}\right),
\end{aligned}
$$

where $L$ is the operator of the Laplace transform and 


$$
\begin{aligned}
\psi\left(t_{1}, t_{2}\right)=\mathrm{L}^{-1} \mathrm{H}_{\nu}^{-1}\left[\frac{\xi^{\nu}}{p^{2}+b^{2} \xi^{2}+c}\right] & \\
(f * \psi)_{\mathrm{L}, \nu}\left(t_{1}, t_{2}\right)= & \frac{t_{2}^{\nu}}{2^{\nu} \sqrt{\pi} \Gamma\left(\nu+\frac{1}{2}\right)} \int_{0}^{t_{1}} d \xi \int_{0}^{\pi} \sin ^{2 \nu} s d s \int_{0}^{\infty} f(\xi, \zeta) \\
& \times \frac{\psi\left(t_{1}-\xi, \sqrt{t_{2}^{2}+\zeta^{2}-2 t_{2} \zeta \cos s}\right)}{\left(t_{2}^{2}+\zeta^{2}-2 t_{2} \zeta \cos s\right)^{\frac{\nu}{2}}} \zeta^{\nu+1} d \zeta, \\
\left(u_{k} * \psi\right)_{\nu}\left(t_{1}, t_{2}\right)= & \frac{t_{2}^{\nu}}{2^{\nu} \sqrt{\pi} \Gamma\left(\nu+\frac{1}{2}\right)} \int_{0}^{\pi} \sin ^{2 \nu} s d s \int_{0}^{\infty} u_{k}(\zeta) \\
& \times \frac{\psi\left(t_{1}, \sqrt{t_{2}^{2}+\zeta^{2}-2 t_{2} \zeta \cos s}\right)}{\left(t_{2}^{2}+\zeta^{2}-2 t_{2} \zeta \cos s\right)^{\frac{\nu}{2}}} \zeta^{\nu+1} d \zeta, \quad k=0,1 .
\end{aligned}
$$

If $c=\gamma^{2}>0, \gamma \in \mathbf{R}$, then

$$
\psi\left(t_{1}, t_{2}\right)=\frac{|\gamma|^{\nu+\frac{1}{2}} t_{2}^{\nu}}{\sqrt{2 \pi}|b|^{\nu+\frac{3}{2}}}\left(b^{2} t_{1}^{2}-t_{2}^{2}\right)^{-\frac{\nu}{2}-\frac{1}{4}} \eta\left(b^{2} t_{1}^{2}-t_{2}^{2}\right) J_{-\nu-\frac{1}{2}}\left(\frac{|\gamma|}{|b|} \sqrt{b^{2} t_{1}^{2}-t_{2}^{2}}\right) .
$$

If $c=0$, then

$$
\psi\left(t_{1}, t_{2}\right)=\frac{2^{\nu} \sqrt{\pi} t_{2}^{\nu}}{|b| \Gamma\left(\frac{1}{2}-\nu\right)}\left(b^{2} t_{1}^{2}-t_{2}^{2}\right)^{-\nu-\frac{1}{2}} \eta\left(b^{2} t_{1}^{2}-t_{2}^{2}\right) .
$$

Example 3. Let $t_{1}, t_{2} \in \mathbf{R}_{+},-1 / 2<\operatorname{Re} \nu<5 / 2$. Then we consider the following equation

$$
\frac{\partial^{2} u}{\partial t_{1}^{2}}+a^{2}\left(\frac{\partial^{2}}{\partial t_{2}^{2}}+\frac{1}{t_{2}} \frac{\partial}{\partial t_{2}}-\frac{\nu^{2}}{t_{2}^{2}}\right)^{2} u\left(t_{1}, t_{2}\right)=f\left(t_{1}, t_{2}\right)
$$

with the conditions

$$
u\left(+0, t_{2}\right)=u_{0}\left(t_{2}\right), \quad u_{t_{1}}\left(+0, t_{2}\right)=u_{1}\left(t_{2}\right) .
$$

The solution of this equation is given by

$$
u\left(t_{1}, t_{2}\right)=(f * \psi)_{\mathrm{L}, \nu}\left(t_{1}, t_{2}\right)+\frac{\partial}{\partial t_{1}}\left(u_{0} * \psi\right)_{\nu}\left(t_{1}, t_{2}\right)+\left(u_{1} * \psi\right)_{\nu}\left(t_{1}, t_{2}\right),
$$

where 


$$
\begin{aligned}
&(f * \psi)_{\mathrm{L}, \nu}\left(t_{1}, t_{2}\right)=\frac{t_{2}^{\nu}}{2^{\nu} \sqrt{\pi} \Gamma\left(\nu+\frac{1}{2}\right)} \int_{0}^{t_{1}} d \xi \int_{0}^{\pi} \sin ^{2 \nu} s d s \int_{0}^{\infty} f(\xi, \zeta) \\
& \times \frac{\psi\left(t_{1}-\xi, \sqrt{t_{2}^{2}+\zeta^{2}-2 t_{2} \zeta \cos s}\right)}{\left(t_{2}^{2}+\zeta^{2}-2 t_{2} \zeta \cos s\right)^{\nu / 2}} \zeta^{\nu+1} d \zeta, \\
&\left(u_{k} * \psi\right)_{\nu}\left(t_{1}, t_{2}\right)=\frac{t_{2}^{\nu}}{2^{\nu} \sqrt{\pi} \Gamma\left(\nu+\frac{1}{2}\right)} \int_{0}^{\pi} \sin ^{2 \nu} s d s \int_{0}^{\infty} u_{k}(\zeta) \\
& \times \frac{\psi\left(t_{1}, \sqrt{t_{2}^{2}+\zeta^{2}-2 t_{2} \zeta \cos s}\right)}{\left(t_{2}^{2}+\zeta^{2}-2 t_{2} \zeta \cos s\right)^{\nu / 2}} \zeta^{\nu+1} d \zeta, \quad k=0,1 ; \\
& \psi\left(t_{1}, t_{2}\right)=\mathrm{L}^{-1} \mathrm{H}_{\nu}^{-1}\left[\xi^{\nu} \Psi(p, \xi)\right]\left(t_{1}, t_{2}\right) \\
&=\frac{t_{2}^{\nu}}{2^{\nu+1}|a|^{\nu+1} t_{1}^{\nu}} \frac{1}{\nu} \sin \frac{\pi \nu}{2}{ }_{1} F_{2}\left(\frac{\nu}{2} ; \frac{1}{2}, \frac{\nu}{2}+1 ;-\frac{t_{2}{ }^{4}}{64 a^{2} t_{1}^{2}}\right) \\
&-\frac{t_{2}{ }^{\nu+2}}{2^{\nu+3}|a|^{\nu+2} t_{1}{ }^{\nu+1}} \frac{1}{\nu+1} \cos \frac{\pi \nu}{2}{ }_{1} F_{2}\left(\frac{\nu+1}{2} ; \frac{3+3}{2} ;-\frac{t_{2}{ }^{4}}{64 a^{2} t_{1}^{2}}\right) .
\end{aligned}
$$

Thus we obtain

$$
\psi\left(t_{1}, t_{2}\right)= \begin{cases}\frac{1}{2|a|} \operatorname{Si}\left(-\frac{t_{2}^{2}}{4|a| t_{1}}\right), & \text { if } \nu=0 \\ \frac{1}{|a| t_{2}} \sin \left(\frac{t_{2}^{2}}{4|a| t_{1}}\right), & \text { if } \nu=1\end{cases}
$$

where

$$
\operatorname{Si}(x)=\int_{0}^{x} \frac{\sin t}{t} d t .
$$

\section{References}

[1] H. Bateman and A. Erdelyi. Tables of Integral Transforms, vol. 2. McGraw-Hill, New York-Toronto-London, 1954.

[2] L.E. Britvina. Poluconvolutions for the Hankel transform and differential operators. Doklady Mathematics, 65(1), 32 - 34, 2002.

[3] L.E. Britvina. Hankel transform belonging to $L\left(\mathbf{R}_{+}\right)$and its application. Vestnik NovSU, 28, $63-66,2004$.

[4] L.E. Britvina. On polyconvolutions generated by the Hankel transform. Mathematical Notes, 76(1), 18 - 24, 2004.

[5] L.E. Britvina. Hankel's polyconvolution with Gegenbauer's polynomial in kernel. Vestnik NovSU, 30, 48 - 51, 2005. 
[6] Yu.A. Brychkov and A.P. Prudnikov. Integral transforms of generalized functions. Nauka, Moscow, 1977. (in Russian)

[7] I.L. Hirschman and D.V. Widder. The convolution transforms. Princeton, New Jersey: Univ. Press, 1955.

[8] V.A. Kakichev. On the convolutions for integral transforms. Izv. Acad. Nauk BSSR. Ser. Fiz.-Mat. Nauk, 22, 48 - 57, 1967.

[9] V.A. Kakichev and L.E. Britvina. On convolutions for the Hankel transform. In: Int. Conference Mathematical Methods in Education, Science, and Industry. Abstracts of Papers, RIO PGU, Tiraspol, 35 - 36, 1999.

[10] A.P. Prudnikov, Yu.A. Brychkov and O.I. Marichev. Integrals and Series. Special Functions. Nauka, Moscow, 1983. (in Russian)

[11] I.N. Sneddon. Fourier Transforms. New York: Dover, 1995.

[12] N.X. Thao and N.T. Xai. Convolution for Integral Transforms and their Applications. Computer Center of the Russian Academy of Sciences, Moscow, 1997.

[13] E.C. Titchmarsh. Introduction to the theory of Fourier integral. Oxford Univ. Press: Oxford, 1937.

[14] G.N. Watson. A treatise on the theory of Bessel function. London, 1945.

[15] S.B. Yakubovich. On the some double convolution type integral transforms. Dokl. AN BSSR, 34(5), 396 - 398, 1990.

[16] Ya.I. Zhitomirskii. The Cauchy problem for systems of linear partial differential equations with differential operators of Bessel type. Mat. Sb. [Math. USSR-Sb.], 36(2), 299 - 310, 1955. 